\title{
Insufficient slow-flow suppression mimicking aneurysm wall enhancement in magnetic resonance vessel wall imaging: a phantom study
}

\author{
Bart M. W. Cornelissen, MSc, ${ }^{1-3}$ Eva L. Leemans, MSc, ${ }^{1,2}$ Bram F. Coolen, PhD, ${ }^{2}$ Eva S. Peper, MSc, ${ }^{1}$ \\ René van den Berg, MD, PhD, ${ }^{1}$ Henk A. Marquering, PhD, ${ }^{1,2}$ Cornelis H. Slump, PhD, ${ }^{3}$ and \\ Charles B. L. M. Majoie, MD, PhD'
}

1Department of Radiology and Nuclear Medicine, Amsterdam Neuroscience, Amsterdam UMC, University of Amsterdam; 2Department of Biomedical Engineering and Physics, Amsterdam UMC, University of Amsterdam; and ${ }^{3}$ MIRA Institute for Biomedical Technology and Technical Medicine, University of Twente, Enschede, The Netherlands

OBJECTIVE MR vessel wall imaging (VWI) is increasingly performed in clinical settings to support treatment decisionmaking regarding intracranial aneurysms. Aneurysm wall enhancement after contrast agent injection is expected to be related to aneurysm instability and rupture status. However, the authors hypothesize that slow-flow artifacts mimic aneurysm wall enhancement. Therefore, in this phantom study they assess the effect of slow flow on wall-like enhancement by using different MR VWI techniques.

METHODS The authors developed an MR-compatible aneurysm phantom model, which was connected to a pump to enable pulsatile inflow conditions. For VWI, 3D turbo spin echo sequences-both with and without motion-sensitized driven equilibrium (MSDE) and delay alternating with nutation for tailored excitation (DANTE) preparation pulses-were performed using a 3-T MR scanner. VWI was acquired both before and after Gd contrast agent administration by using two different pulsatile inflow conditions $(2.5 \mathrm{ml} / \mathrm{sec}$ peak flow at 77 and 48 beats per minute). The intraluminal signal intensity along the aneurysm wall was analyzed to assess the performance of slow-flow suppression.

RESULTS The authors observed wall-like enhancement after contrast agent injection, especially in low pump rate settings. Preparation pulses, in particular the DANTE technique, improved the performance of slow-flow suppression.

CONCLUSIONS Near-wall slow flow mimics wall enhancement in VWI protocols. Therefore, VWI should be carefully interpreted. Preparation pulses improve slow-flow suppression, and therefore the authors encourage further development and clinical implementation of these techniques.

https://thejns.org/doi/abs/10.3171/2019.4.FOCUS19235

KEYWORDS intracranial aneurysms; vessel wall imaging; slow flow

$\mathrm{F}$ loR patients with multiple aneurysms and an acute hemorrhagic stroke, it is important to identify the ruptured aneurysm. Aneurysm rupture status cannot always be determined based on the bleeding pattern alone. Aneurysm size ${ }^{4}$ and morphology ${ }^{2}$ can help in decisionmaking, but additional characteristics are required to improve the identification of ruptured aneurysms.

\section{Vessel Wall Imaging}

Aneurysm wall assessment using MR vessel wall imaging (VWI) is increasingly performed to identify ruptured or unstable aneurysms..11,12 MR VWI enables aneurysm wall assessment by suppressing MR signal originating from flowing blood and CSF. 3D turbo spin echo (TSE) is a commonly used VWI technique, which mainly suppresses flow in the readout direction. ${ }^{14}$ Because intracranial blood flow is often complex (slow, stagnant, or turbulent), preparation pulses have been developed to optimize flow suppression. Commonly used preparation pulses are motionsensitized driven equilibrium (MSDE) and delay alternating with nutation for tailored excitation (DANTE), ${ }^{7,17,18}$

Aneurysm wall enhancement after Gd contrast agent injection is suggested to be related to inflammation in

ABBREVIATIONS bpm = beats per minute; DANTE = delay alternating with nutation for tailored excitation; MCA = middle cerebral artery; MSDE = motion-sensitized driven equilibrium; TSE = turbo spin echo; VENC = velocity encoding; VWI = vessel wall imaging; WSS = wall shear stress.

SUBMITTED March 5, 2019. ACCEPTED April 23, 2019.

INCLUDE WHEN CITING DOI: 10.3171/2019.4.FOCUS19235. 
unstable aneurysm walls. ${ }^{6}$ However, the performance of blood suppression in VWI decreases after Gd contrast agent injection, because $\mathrm{Gd}$ shortens the $\mathrm{T} 1$ relaxation time of blood. A hyperintense appearance originating from unsuppressed blood could therefore potentially be mistaken for aneurysm wall, which is an important pitfall of MR VWI. ${ }^{5,8}$ The aim of this phantom study was to assess the contribution of slow flow to wall-like enhancement in 3D TSE VWI, both with and without MSDE and DANTE preparation pulses.

\section{Methods}

\section{Phantom Development}

We developed an MR-compatible aneurysm phantom model based on a stable middle cerebral artery (MCA) aneurysm $(6 \mathrm{~mm})$ from a patient who was studied with 7-T MR VWI (Fig. 1) The 7-T time-of-flight MR angiography data were used to segment the aneurysm and perianeurysmal vasculature by using a level set segmentation algorithm from the Vascular Modeling Toolkit (http:// www.vmtk.org). Subsequently, the 3 outflow vessels were manually connected using open-source 3D modeling software (Blender version 2.74, http://www.blender.org/). A 3D printer (Connex3 Objet260; Stratasys) was used to print a hollow vessel lumen in a solid plastic box. VeroClear, a transparent polymer, was used as printing material. The benefit of such a polymer is that it does not get magnetized during the MRI session, and therefore appears black in VWI. Finally, inflow and outflow tubes were connected to the 3D-printed model, and then the model was submerged in an agar solution to obtain a homogeneous local magnetic field. Figure 2 demonstrates the phantom development pipeline.

\section{MRI Experiments}

In Fig. 3, a schematic overview of our MR phantom experiment setup is presented. MRI acquisitions were performed using a 3-T Ingenia MR scanner (Philips Healthcare) and a 32-channel head coil. The phantom was connected to a pump outside the scanner room to generate pulsatile inflow conditions. We used water to pump through the phantom model and performed $2 \mathrm{D}$ phase-contrast MRI (resolution $0.38 \times 0.38 \times 3 \mathrm{~mm}$; TE/TR 6.9/15.7 msec; flip
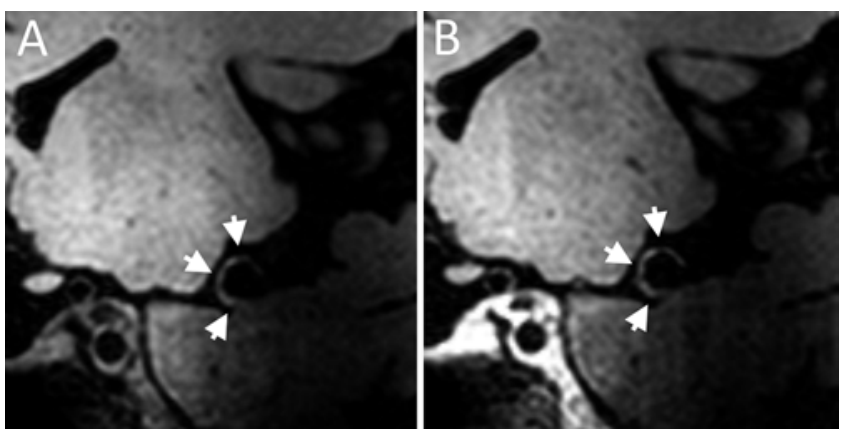

FIG. 1. 7-T MR VWI before (A) and after (B) contrast agent administration, acquired in a patient harboring a stable MCA aneurysm. The white arrows indicate the aneurysm wall.
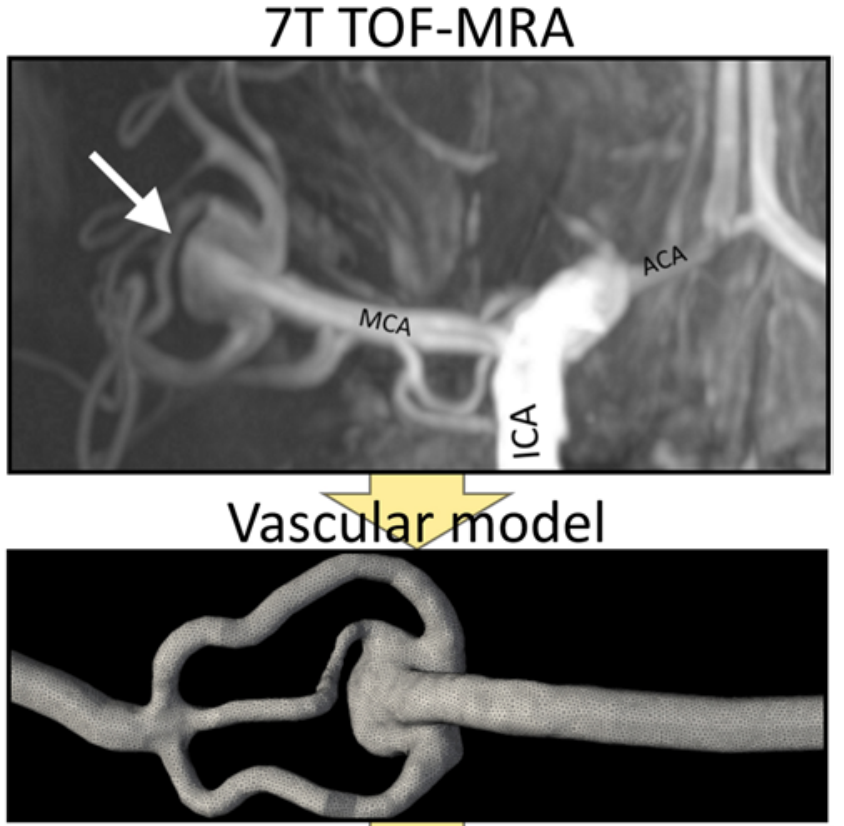

3D print

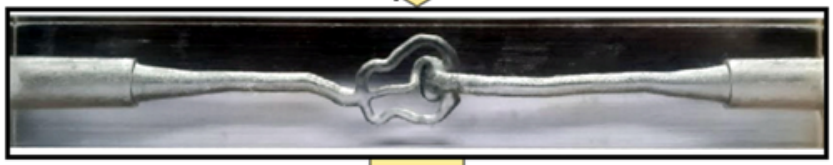

\section{MR-phantom}

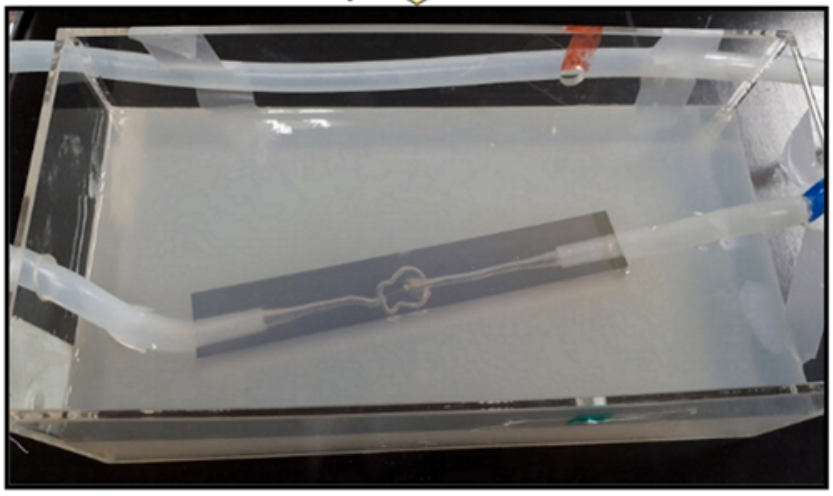

FIG. 2. Phantom model development pipeline, based on 7-T time-offlight (TOF) MR angiography patient data. Level set segmentation was performed to isolate the aneurysm (white arrow) and inflow/outflow vessels. The outflow vessels were connected using 3D modeling software. The vascular model was 3D printed as a hollow vessel lumen in a solid plastic box. To finalize the phantom, in- and outflow tubes were connected to the 3D printed model, and then the model was submerged in an agar solution. $\mathrm{ACA}=$ anterior cerebral artery; $\mathrm{ICA}=$ internal carotid artery.

angle $25^{\circ}$; velocity encoding [VENC] $80 \mathrm{~cm} / \mathrm{sec} ; 26$ cardiac phases) perpendicular to the parent vessel to measure the inflow waveform. For pulsatile inflow conditions, we generated a peak flow of approximately $2.5 \mathrm{ml} / \mathrm{sec}$, which is comparable to physiological MCA inflow. ${ }^{3}$ Pump rates of 77 and 48 beats per minute (bpm) were used to simulate different intra-aneurysmal flow conditions: peak flow ve- 


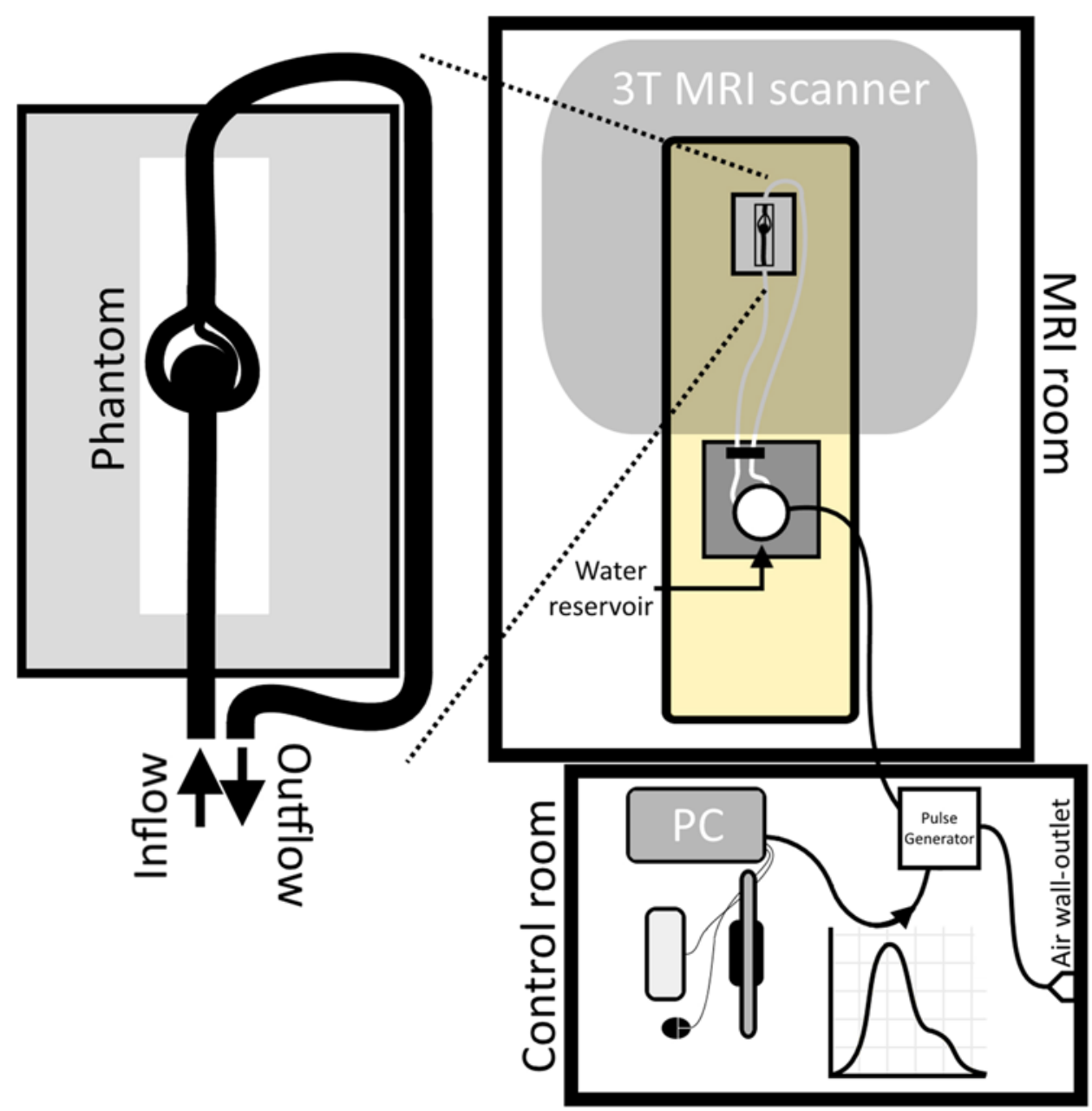

FIG. 3. Schematic overview of the MR experiment setup. The phantom and pump reservoir were situated in the MRI room. The reservoir was connected to the pump in the control room, which was controlled by a desktop computer (PC) to generate pulsatile inflow conditions.

locities remained similar, but average velocities were different in both settings.

VWI was performed before and after Gd (gadobutrol, Gadavist; Bayer BV) enhancement. Based on the drug description, gadobutrol is rapidly distributed in plasma: 2 minutes after administration the in vivo plasma concentration is $0.59 \mathrm{mmol} / \mathrm{L}, 60$ minutes after administration the plasma concentration is $0.3 \mathrm{mmol} / \mathrm{L}$. Assuming exponential distribution dynamics and VWI acquisition 15 minutes after venous contrast injection, we estimate the concentration to be approximately $0.4 \mathrm{mmol}$ gadobutrol per liter of plasma in clinical settings. Therefore, for our contrast-enhanced phantom experiments we used this concentration.

Finally, we acquired a 4D flow scan (TE/TR 8.1/4.5 $\mathrm{msec}$; VENC $80 \mathrm{~cm} / \mathrm{sec}$; spatial resolution $0.5 \times 0.5 \times 0.5$ $\mathrm{mm} ; 10$ cardiac phases) to analyze the relationship between flow suppression and flow velocity. The 4D flow MR scan was acquired with an acceleration factor of 5 in combination with a compressed sensing reconstruction strategy in order to speed up the scan time. ${ }^{13}$

\section{D TSE VWI Techniques}

We acquired 3D TSE VWI scans with and without MSDE and DANTE preparation pulses, at an isotropic resolution of $0.7 \mathrm{~mm}$. All VWI acquisitions used the same variable flip angle scheme and a field of view of $320 \times$ $320 \times 140 \mathrm{~mm}$. The TRs were $700 \mathrm{msec}$ for 3D TSE and MSDE-prepared VWI, and $1000 \mathrm{msec}$ for the DANTEprepared VWI. It is important to note that pump rates of 77 and $48 \mathrm{bpm}$ were chosen to ensure VWI acquisition throughout the entire pulsatile cycle. Pump frequencies synchronized with VWI TRs had resulted in unreliable behavior of flow-suppressing performance in previous experiments (e.g., pump frequency of $1 \mathrm{~Hz}[60 \mathrm{bpm}]$ with VWI repetition times of $1000 \mathrm{msec}$ ).

\section{Analyses}

A mask along the aneurysm wall was created to analyze near-wall signal intensities in VWI (Fig. 4). Additionally, a mask was created for a static agar region. Near-wall signal intensities were normalized to the mean agar intensity 


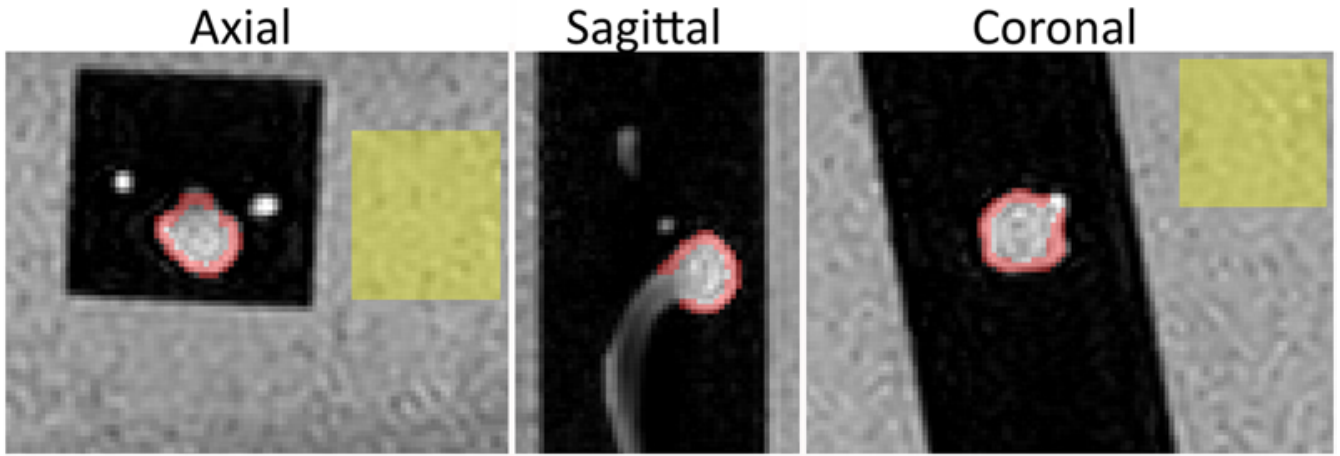

FIG. 4. Masks for signal intensity calculations. The intraluminal mask along the aneurysm wall is shown in red. The agar mask is shown in yellow. To calculate the relative intensity, signal intensities along the aneurysm wall were normalized to the average intensity of the masked agar tissue.

to make them comparable between VWI sequences. Box plots and histograms of normalized signal intensities were visually compared between pre- and postcontrast VWI, separately for each VWI technique and pump setting.

\section{Results}

Signals originating from slow flow along the aneurysm wall were observed for all VWI techniques and became more apparent after contrast enhancement, especially at lower pump rates (Fig. 5). Visual assessment of VWI suggested that preparation pulses improved flow suppression. Histograms and box plots of normalized signal intensities along the aneurysm wall demonstrated clear differences between VWI techniques (Fig. 6). After MSDE preparation, flow suppression improved. A larger effect on flow suppression improvement was observed for DANTE preparation. Near-wall signal intensities decreased by approxi-

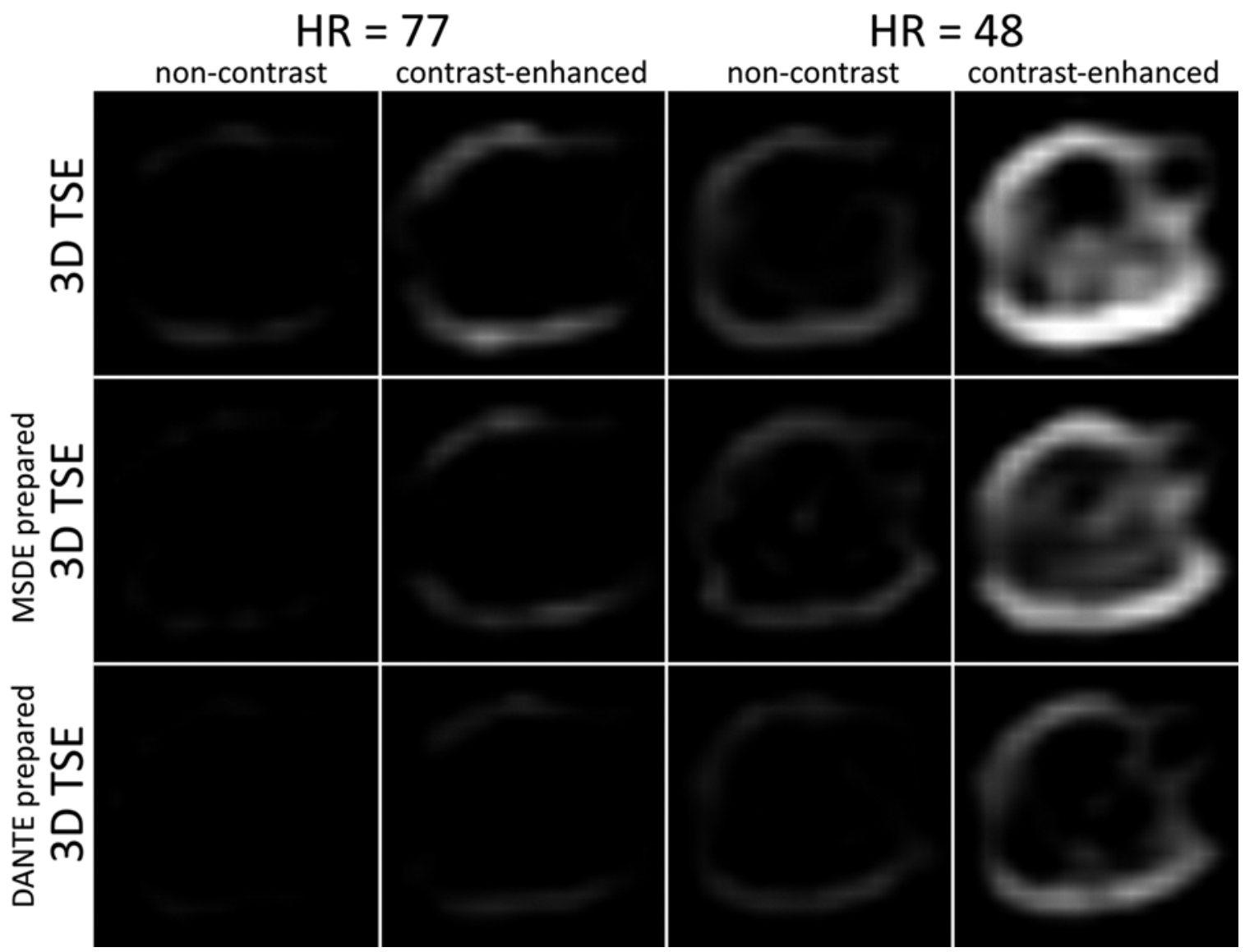

FIG. 5. VWI before and after contrast enhancement for different VWI techniques (rows) and inflow settings (columns). Visual assessment suggests that signal intensities are higher in contrast-enhanced groups and at a lower pump rate. $H R=$ heart rate. 


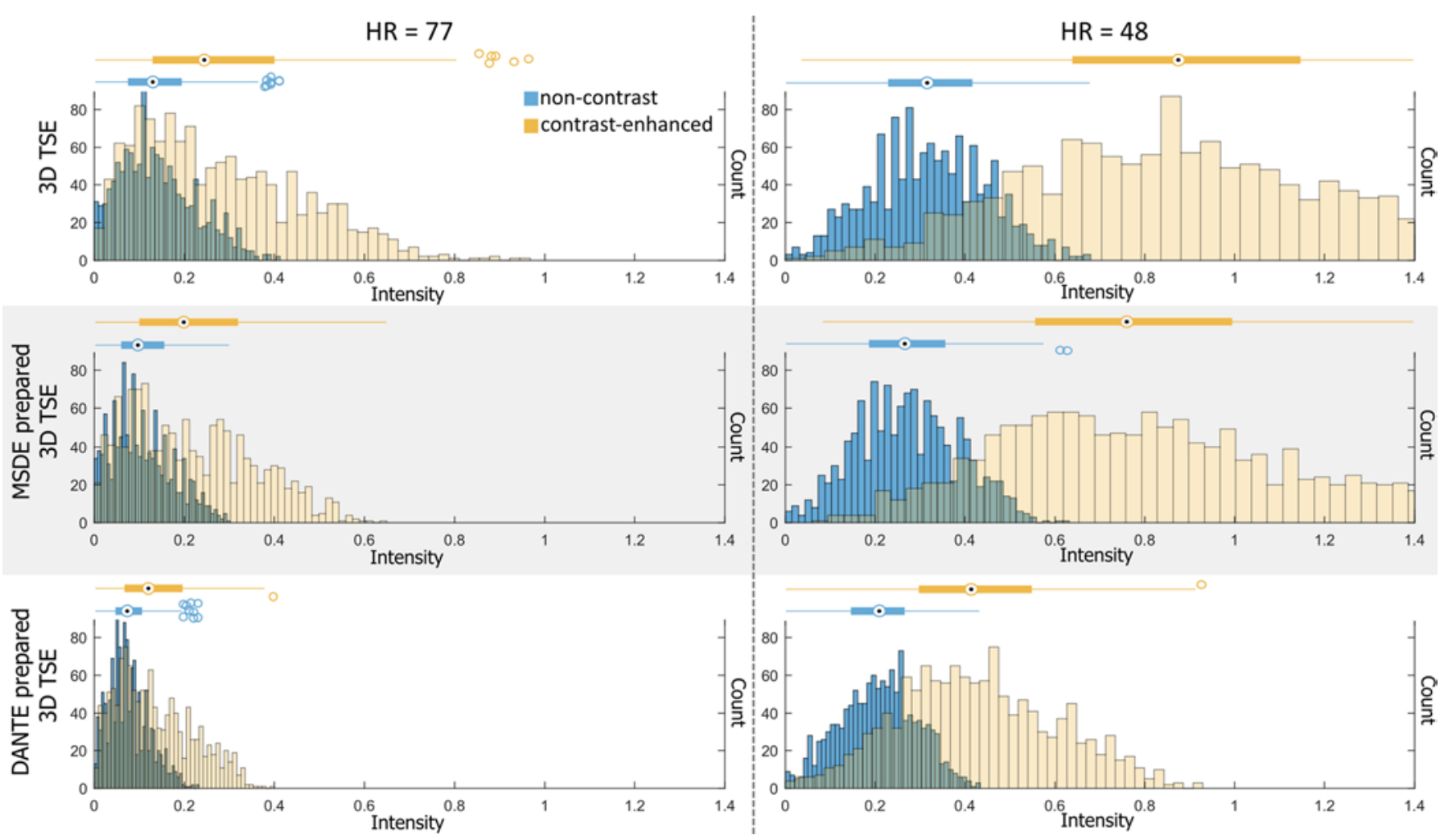

FIG. 6. Histograms and box plots (blue and yellow horizontal bars) of the near-wall intraluminal signal intensity, normalized to the surrounding agar intensity. Different VWI techniques are presented in each row, and each column represents a different inflow condition. The histograms are transparent; gray vertical bars show the overlap between VWI studies obtained with and without contrast.

mately $50 \%$ after DANTE preparation, compared to 3D TSE VWI alone. Figure 7 shows a comparison between contrast-enhanced 3D TSE VWI and 4D flow imaging for the same slice location. The region of high flow velocity clearly corresponds to the dark, suppressed region in VWI. This confirms the relationship between flow and suppression.

\section{Discussion}

In our phantom model, we observed wall-like MR signal along the aneurysm wall in VWI both with and without preparation pulses. After contrast agent administration, these signals became more apparent, especially at low pump rates. Signal originating from unsuppressed blood may easily be mistaken for aneurysm wall. Therefore, we underline the importance of careful interpretation of VWI. Preparation pulses, especially the DANTE technique, improved flow suppression.

\section{Contribution of Slow Flow to Enhancement in VWI}

To our knowledge, this is the first analysis of flowrelated artifacts in VWI in a controlled phantom setup. However, the contribution of slow flow to aneurysm wall enhancement has been assessed in patients harboring unruptured intracranial aneurysms by comparing VWI with and without MSDE preparation pulses. ${ }^{5}$ Wall enhancement was observed less often in MSDE-prepared VWI, suggesting wall-mimicking enhancement on conventional VWI without preparation pulses. Comparably, in our study we showed lower signal intensities along the wall for MSDE-prepared VWI, compared to VWI without a preparation pulse. Another study emphasized that slow-flow artifacts, which are most obvious in slow-flowing blood in veins, may result in misinterpretation of enhancement of surrounding arterial vessel walls. ${ }^{8}$ Similarly, we observed slow-flow artifacts in slow-flowing blood in the aneurysm sac, which may easily be misinterpreted as aneurysm wall enhancement.

\section{Traditional Risk Factors (Lesion Size and Shape) are Sensitive to Contribution of Slow Flow in VWI}

Various studies have presented relationships between aneurysm wall enhancement and aneurysm instability, often neglecting the potential influence of slow flow on wall assessment. Aneurysm wall enhancement has been observed at irregular regions and daughter sacs of aneurysms. ${ }^{16}$ Because slow and turbulent flow characteristics are expected in irregular aneurysm regions, flow suppression could have been hindered, resulting in wall-like enhancement. Similarly, several studies have shown a relationship between wall enhancement and aneurysm size., ${ }^{1,910,20}$ However, larger aneurysms are most likely also to be associated with lower flow velocities, which reduces flow suppression performance. Furthermore, regions of low wall shear stress (WSS) have been related to wall enhancement. ${ }^{19}$ Because 

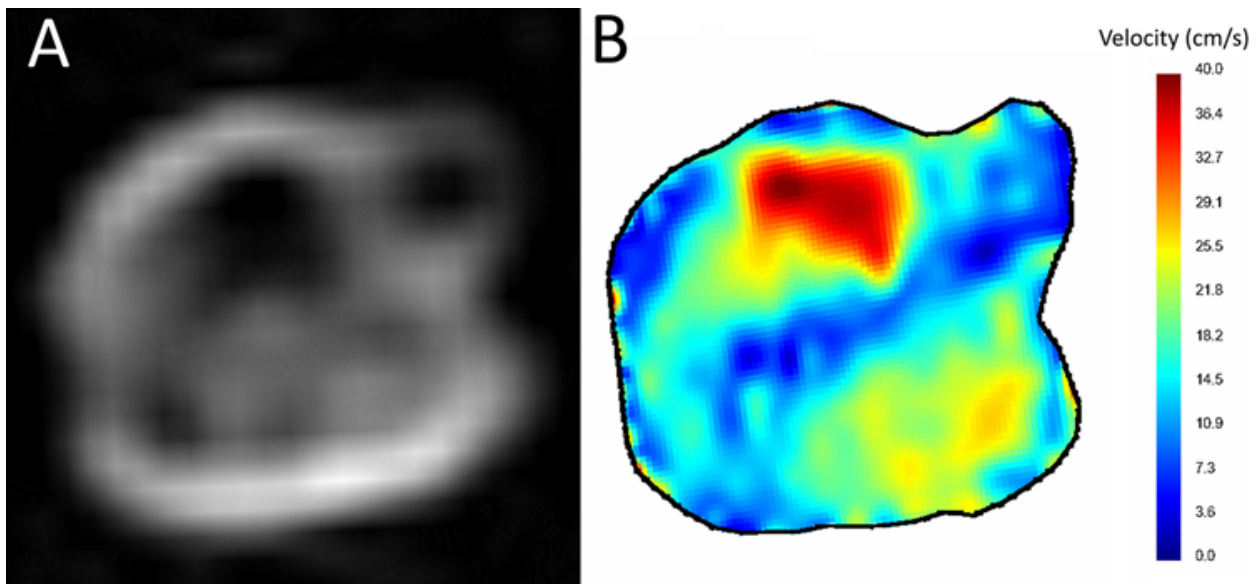

FIG. 7. A comparison between contrast-enhanced 3D TSE VWI at $48 \mathrm{bpm}(\mathrm{A})$ and 4D flow imaging (B) at the same location at 60 $\mathrm{bpm}$. The region of high flow velocity clearly corresponds to the dark region in VWI. This confirms the relationship between suppression and flow.

low WSS is related to lower velocities near the wall, insufficient suppression of slow flow in VWI could have contributed to wall enhancement. Putting it into perspective, slow flow contributes to low WSS, which has been suggested to be related to inflammation in the aneurysm wall..$^{5}$ Therefore, in enhanced unstable aneurysms, enhancement is most likely to be caused by a combination of slow flow and inflammation in the aneurysm wall.

\section{Study Limitations}

Our study has several limitations. We created pulsatile inflow conditions comparable to physiological MCA peak flow rates. However, we could not recreate physiological diastolic flow rates (approximately $1.5 \mathrm{ml} / \mathrm{sec}$ at late diastole) by using the MR-compatible pump. Instead, no flow was present in late diastole. This may have resulted in an overestimation of unsuppressed flow signal when compared to an in vivo situation. In addition, we modeled different flow velocities by using different pump rates with similar peak flows. In vivo, not only heart rate, but also stroke volume, hypertension, and other hemodynamic parameters may affect local flow velocities. However, our study indicates that in regions with slow flow, insufficient flow suppression mimics aneurysm wall enhancement. Another limitation is the rigid design of our phantom model, which does not allow pulsatile behavior along the wall. Arterial compliance probably results in higher blood flow velocities along the vessel wall compared to our phantom model. This may have resulted in a higher chance of false-positive enhancement. However, we believe that slow and recirculating flow characteristics are inherently related with intracranial aneurysms that-depending on aneurysm size and shapemay contribute to wall-mimicking enhancement. Finally, we used water instead of blood for our experiments. Water lacks the non-newtonian behavior of blood and, more importantly, it has a lower viscosity compared to blood. Lower flow velocities near the vessel wall can be expected in more viscous fluids. In theory, flow suppression would have been worse when using a higher-viscosity fluid in our phantom setup.

\section{Conclusions}

Insufficient slow-flow suppression mimics aneurysm wall enhancement in MR VWI. Therefore, vessel wall imaging should be carefully interpreted. Preparation pulses improve slow-flow suppression; therefore, we encourage further development and clinical implementation of these flow suppression techniques.

\section{Acknowledgments}

An unrestricted grant was provided to Amsterdam University Medical Centers, Academic Medical Center, by the foundation Toegepast Wetenschappelijk Instituut voor Neuromodulatie (TWIN), The Netherlands. The TWIN foundation had no role in study design, data collection, analysis or interpretation, or the writing of the report. The corresponding author had full access to all the data in the study and had final responsibility for the decision to submit for publication.

We thank H. Kuipers and G. A. te Riet og Scholten for 3D printing of the phantom model. Furthermore, we would like to acknowledge G. Gilbert, PhD, and J. C. W. Siero, PhD, for facilitating the DANTE implementation.

\section{References}

1. Backes D, Hendrikse J, van der Schaaf I, Algra A, Lindgren $\mathrm{AE}$, Verweij BH, et al: Determinants of gadolinium-enhancement of the aneurysm wall in unruptured intracranial aneurysms. Neurosurgery 83:719-725, 2018

2. Backes D, Vergouwen MDI, Velthuis BK, van der Schaaf IC, Bor AS, Algra A, et al: Difference in aneurysm characteristics between ruptured and unruptured aneurysms in patients with multiple intracranial aneurysms. Stroke 45:1299-1303, 2014

3. Cornelissen BMW, Schneiders JJ, Sprengers ME, van den Berg R, van Ooij P, Nederveen AJ, et al: Aneurysmal parent artery-specific inflow conditions for complete and incomplete circle of Willis configurations. AJNR Am J Neuroradiol 39:910-915, 2018

4. Greving JP, Wermer MJH, Brown RD Jr, Morita A, Juvela $\mathrm{S}$, Yonekura M, et al: Development of the PHASES score for prediction of risk of rupture of intracranial aneurysms: a pooled analysis of six prospective cohort studies. Lancet Neurol 13:59-66, 2014 
5. Kalsoum E, Chabernaud Negrier A, Tuilier T, Benaïssa A, Blanc R, Gallas S, et al: Blood flow mimicking aneurysmal wall enhancement: A diagnostic pitfall of vessel wall MRI using the postcontrast 3D turbo spin-echo MR imaging sequence. AJNR Am J Neuroradiol 39:1065-1067, 2018

6. Larsen N, von der Brelie C, Trick D, Riedel CH, Lindner T, Madjidyar J, et al: Vessel wall enhancement in unruptured intracranial aneurysms: an indicator for higher risk of rupture? High-resolution MR imaging and correlated histologic findings. AJNR Am J Neuroradiol 39:1617-1621, 2018

7. Li L, Miller KL, Jezzard P: DANTE-prepared pulse trains: a novel approach to motion-sensitized and motion-suppressed quantitative magnetic resonance imaging. Magn Reson Med 68:1423-1438, 2012

8. Lindenholz A, van der Kolk AG, Zwanenburg JJM, Hendrikse J: The use and pitfalls of intracranial vessel wall imaging: how we do it. Radiology 286:12-28, 2018

9. Liu P, Qi H, Liu A, Lv X, Jiang Y, Zhao X, et al: Relationship between aneurysm wall enhancement and conventional risk factors in patients with unruptured intracranial aneurysms: a black-blood MRI study. Interv Neuroradiol 22:501-505, 2016

10. Lv N, Karmonik C, Chen S, Wang X, Fang Y, Huang Q, et al: Relationship between aneurysm wall enhancement in vessel wall magnetic resonance imaging and rupture risk of unruptured intracranial aneurysms. Neurosurgery [epub ahead of print], 2018

11. Matouk CC, Mandell DM, Günel M, Bulsara KR, Malhotra A, Hebert R, et al: Vessel wall magnetic resonance imaging identifies the site of rupture in patients with multiple intracranial aneurysms: proof of principle. Neurosurgery 72:492-496, 2013

12. Omodaka S, Endo H, Niizuma K, Fujimura M, Inoue T, Sato $\mathrm{K}$, et al: Quantitative assessment of circumferential enhancement along the wall of cerebral aneurysms using MR imaging. AJNR Am J Neuroradiol 37:1262-1266, 2016

13. Peper ES, Gottwald LM, Zhang Q, Coolen BF, van Ooij P, Strijkers GJ, et al: 30 times accelerated 4D flow MRI in the carotids using a pseudo spiral Cartesian acquisition and a total variation constrained compressed sensing reconstruction. Proc Intl Soc Magn Reson Med Sci Meet Exhib 26:1-3, 2018

14. Qiao Y, Steinman DA, Qin Q, Etesami M, Schär M, Astor $\mathrm{BC}$, et al: Intracranial arterial wall imaging using threedimensional high isotropic resolution black blood MRI at 3.0 Tesla. J Magn Reson Imaging 34:22-30, 2011
15. Tulamo R, Frösen J, Hernesniemi J, Niemelä M: Inflammatory changes in the aneurysm wall: a review. J Neurointerv Surg 2:120-130, 2010

16. Wang GX, Wen L, Lei S, Ran Q, Yin JB, Gong ZL, et al: Wall enhancement ratio and partial wall enhancement on MRI associated with the rupture of intracranial aneurysms. J Neurointerv Surg 10:566-570, 2018

17. Wang J, Helle M, Zhou Z, Börnert P, Hatsukami TS, Yuan C: Joint blood and cerebrospinal fluid suppression for intracranial vessel wall MRI. Magn Reson Med 75:831-838, 2016

18. Wang J, Yarnykh VL, Yuan C: Enhanced image quality in black-blood MRI using the improved motion-sensitized driven-equilibrium (iMSDE) sequence. J Magn Reson Imaging 31:1256-1263, 2010

19. Xiao W, Qi T, He S, Li Z, Ou S, Zhang G, et al: Low wall shear stress is associated with local aneurysm wall enhancement on high-resolution MR vessel wall imaging. AJNR Am J Neuroradiol 39:2082-2087, 2018

20. Zhu C, Wang X, Degnan AJ, Shi Z, Tian B, Liu Q, et al: Wall enhancement of intracranial unruptured aneurysm is associated with increased rupture risk and traditional risk factors. Eur Radiol 28:5019-5026, 2018

\section{Disclosures}

The authors report no conflict of interest concerning the materials or methods used in this study or the findings specified in this paper.

\section{Author Contributions}

Conception and design: all authors. Acquisition of data: Cornelissen, Leemans, Coolen, Peper. Analysis and interpretation of data: all authors. Drafting the article: Cornelissen. Critically revising the article: Leemans, Coolen, Peper, van den Berg, Marquering, Slump, Majoie.

\section{Correspondence}

Bart M. W. Cornelissen: Amsterdam UMC, Amsterdam, The Netherlands.b.m.cornelissen@amc.uva.nl. 